\title{
Synthesis, Structure, and Characterization of Keggin-Type Germanate
}

\author{
Ya-feng Li, ${ }^{1,2}$ Xiao-lin Qin, ${ }^{1}$ Yue Xu, ${ }^{1}$ Wen-yuan Gao, ${ }^{1}$ and Yue Gao ${ }^{1}$ \\ ${ }^{1}$ School of Chemical Engineering, Changchun University of Technology, Changchun 130012, China \\ ${ }^{2}$ College of Chemistry, Jilin University, Changchun 130023, China \\ Correspondence should be addressed to Ya-feng Li; fly012345@sohu.com
}

Received 13 June 2012; Revised 20 September 2012; Accepted 18 October 2012

Academic Editor: Cengiz Soykan

Copyright (c) 2013 Ya-feng Li et al. This is an open access article distributed under the Creative Commons Attribution License, which permits unrestricted use, distribution, and reproduction in any medium, provided the original work is properly cited.

\begin{abstract}
A novel Keggin-type germanate, $\left(\mathrm{NH}_{4}\right)_{9}\left[\mathrm{Ge}_{7} \mathrm{O}_{14} \mathrm{~F}_{3}\right]_{3} \cdot 1.75 \mathrm{H}_{2} \mathrm{O}(\mathrm{I})$, is hydrothermally synthesized and structurally characterized by X-ray single-crystal diffraction, elemental analysis, XRD, and TG. (I) is tetragonal system with space group I4/mmm and unit cell: $a=28.280$ (4) $\AA, c=24.881(5) \AA, V=19899(6) \AA^{3}, D c=1.632 \mathrm{~g} / \mathrm{cm}^{3}, \mu(\mathrm{Mo} \mathrm{K} \alpha)=6.309 \mathrm{~mm}^{-1}, Z=2, R_{1}=0.1141$ for 2576 reflections with $\mathrm{Fo}>2(\mathrm{Fo})$. $\mathrm{Ge}_{7} \mathrm{O}_{14} \mathrm{~F}_{3}$ entry is defined as the cluster including one octahedron, two edge-sharing triganol bipyramids, and four tetrahedra. Every $\mathrm{Ge}_{7} \mathrm{O}_{14} \mathrm{~F}_{3}$ entry links adjacent four $\mathrm{Ge}_{7} \mathrm{O}_{14} \mathrm{~F}_{3}$ entries by four tetrahedra. Twelve $\mathrm{Ge}_{7} \mathrm{O}_{14} \mathrm{~F}_{3}$ entries construct a cage with all octahedra of $\mathrm{Ge}_{7} \mathrm{O}_{14} \mathrm{~F}_{3}$ pointing inside, which can be simplified into Keggin-type cage through $\mathrm{Ge}_{7} \mathrm{O}_{14} \mathrm{~F}_{3}$ as the node. The solvent experiment proves that (I) is stable in the water and sensitive to base and acid. The result of XRD shows that the structural water of (I) is easily lost to drop the crystalline. The thermal study indicates that the Keggin-type cage of (I) begins to partly collapse at $200^{\circ} \mathrm{C}$ and finally changes into $\mathrm{GeO}_{2}$.
\end{abstract}

\section{Introduction}

Over the two decades, more efforts have been focused on synthesis of germanates because germanates could not only form the zeolites or molecular sieves $[1,2]$ but also achieve more openness than silicates owing to smaller rings and lower framework density based on the flexible $\mathrm{Ge}-\mathrm{O}-\mathrm{Ge}$ of $\sim 130^{\circ}[3-5]$. It is hard for germanium to condense itself into zeolite due to the limitation of synthesis method. Only several examples have been found as BEC, ASV, and UOZ [6-8], and more instances can be accessible by germanium and the other tetrahedral elements- $\mathrm{B}, \mathrm{Al}, \mathrm{Ga}, \mathrm{Si}$, and so on [5, 9-17]. As a result of the large atomic radii of germanium conforms higher five- and six-coordination rather than four-coordination, the clusters comprised of the mixed coordinations give rise to large porous and extra porous frameworks [18-23]. The mixed 4-, 5- and 6-coordination $\mathrm{Ge}_{7} \mathrm{O}_{14} \mathrm{~F}_{3}$ cluster is discussed in detail as robust building unit of $2 \mathrm{D}$ and $3 \mathrm{D}$ nets [24-27]. In recently reported tubular germanate [22], $\left[\left(\mathrm{C}_{5} \mathrm{~N}_{2} \mathrm{H}_{14}\right)_{4}\left(\mathrm{C}_{5} \mathrm{~N}_{2} \mathrm{H}_{13}\right)\left(\mathrm{H}_{2} \mathrm{O}\right)_{4}\right]$ $\left[\mathrm{Ge}_{7} \mathrm{O}_{12} \mathrm{O}_{4 / 2}(\mathrm{OH}) \mathrm{F}_{2}\right]\left[\mathrm{Ge}_{7} \mathrm{O}_{12} \mathrm{O}_{5 / 2}(\mathrm{OH}) \mathrm{F}_{2}\left[\mathrm{GeO}_{2 / 2}(\mathrm{OH})_{2}\right]\right.$, twelve $\mathrm{Ge}_{7} \mathrm{O}_{14} \mathrm{~F}_{3}$ clusters form a cage which mimics the Keggin structure with respect to $\mathrm{Ge}_{7} \mathrm{O}_{14} \mathrm{~F}_{3}$ cluster as the node. Interestingly, this Keggin-type germanate cage possesses $5.3 \AA$ aperture and $8.3 \AA$ cavity, which is bigger than Keggin-type POM- $\mathrm{PMo}_{12} \mathrm{O}_{40}{ }^{3-}$. In this work, we have aimed to crystallize Keggin-type germanate, $\left(\mathrm{NH}_{4}\right)_{9}\left[\mathrm{Ge}_{7} \mathrm{O}_{14} \mathrm{~F}_{3}\right]_{3} \cdot 1.75 \mathrm{H}_{2} \mathrm{O}$-CCUT-8 (denoted as the Changchun University of Technology) and furthermore studied the solvent and thermal stabilities of Keggin-type cage.

\section{Experimental Section}

2.1. Materials and Instrument. All of the reagents were of analytical grade and used as received. The deionized water was used in all the experiments.

The infrared (IR) spectra were recorded within the 400 $4000 \mathrm{~cm}^{-1}$ region on a BRUKER Vertex 70 FTIR spectrometer using $\mathrm{KBr}$ pellets. The elemental analyses were performed on 
a PerkinElmer 2400 element analyzer. Powder X-ray diffraction (XRD) patterns were recorded on a Rigaku D/MAX PC2200 diffractometer for $\mathrm{Cu} K \alpha$ radiation $(\lambda=1.5406 \AA)$, with a scan speed of $5^{\circ} / \mathrm{min}^{-1}$. The thermal gravimetric analyses (TG) were performed on Pyris Diamond TG/DTA instrument used in an atmospheric environment with a heating rate of $10^{\circ} \mathrm{C} / \mathrm{min}$.

2.2. Synthesis. The colorless crystals of $\left(\mathrm{NH}_{4}\right)_{9}\left[\mathrm{Ge}_{7} \mathrm{O}_{14} \mathrm{~F}_{3}\right]_{3} \cdot 1.75 \mathrm{H}_{2} \mathrm{O}$ were obtained under solvothermal condition. $\mathrm{GeO}_{2}(0.25 \mathrm{~g})$ was firstly dispersed in $\mathrm{H}_{2} \mathrm{O}(1 \mathrm{~mL})$. Then pyridine $(4 \mathrm{~mL}), 2$-methylpiperazine $(0.95 \mathrm{~g})$, and hydrofluric acid $(0.5 \mathrm{~mL})$ were successively added under vigorous stirring. The clear solution with molar ratio of $1 \mathrm{GeO}_{2}: 4$ (2-methylpiperazine): 50 pyridine: 58.3 $\mathrm{H}_{2} \mathrm{O}: 4.8 \mathrm{HF}$ was stirring for 4 hours and then it was transferred into $15 \mathrm{~mL}$ stainless steel autoclave and heated at $438 \mathrm{~K}$ for 14 days. After naturally cooled to room temperature, colorless product was collected as a single phase. All the crystals were washed by water and alcohol. The resultant crystals were dried naturally before single-crystal

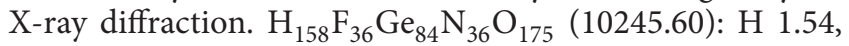
$\mathrm{N}$ 4.92; found $\mathrm{H} 1.74, \mathrm{~N} 5.32$.

2.3. Spectra of FTIR. The peaks at 3254 and $1590 \mathrm{~cm}^{-1}$ were attributed to the stretching and bending vibrations of $\mathrm{NH}_{4}^{+}$; the peaks at 3446 and $1454 \mathrm{~cm}^{-1}$ were assigned to stretching and bending vibrations of $\mathrm{H}_{2} \mathrm{O}$; the peaks at $860,831,578$, $460 \mathrm{~cm}^{-1}$ were due to vibrations of $\mathrm{Ge}-\mathrm{O}$ or $\mathrm{Ge}-\mathrm{F}$.

2.4. Thermal Stability. The results of thermal gravimetric analyses showed that the total weight loss was $15.3 \%$ from room temperature to $600^{\circ} \mathrm{C}$, corresponding to structural water and decomposition of ammonia and fluoride (calculated value: $15.6 \%$ ), respectively, (Figure 1). CCUT-8 experienced the two weight losses from room temperature to $600^{\circ} \mathrm{C}$ and gave rise to final residues $\left(\mathrm{GeO}_{2}\right)$. The gradual part from $100^{\circ} \mathrm{C}$ to $250^{\circ} \mathrm{C}$ was assigned to structural water, and the sharp part from $300^{\circ} \mathrm{C}$ to $350^{\circ} \mathrm{C}$ was attributed to the decomposition of ammonia and fluoride.

The crystalline and thermal stability were investigated through the XRD (Figure 2). The broad and weak peak of (I) in XRD pattern of room temperature indicated that the crystalline of (I) decreased owing to fast loss of the structure water. Furthermore, we made an attempt to fuse the separated Keggin-type cage of (I) by condensing the oxygen of adjacent cage to build the solid structure. However, the XRD of the samples treated at $200^{\circ} \mathrm{C}$ and $300^{\circ} \mathrm{C}$ for $3 \mathrm{hrs}$ in the air showed that Keggin-type cage of (I) began to partly collapse at $200^{\circ} \mathrm{C}$ and basically changed into the $\mathrm{GeO}_{2}$ at $300^{\circ} \mathrm{C}$, which consisted with the results of TG.

2.5. X-Ray Single-Crystal Diffraction. X-ray diffraction data were collected at $293 \mathrm{~K}$ from a suitable single crystal sealed in glass capillary on a Rigaku R-AXIS RAPID diffractometer equipped with graphite-monochromatized Mo K $\alpha$ radiation $(\lambda=0.71073 \AA)$. The structure was solved by the directmethod routine of SHELXS-97 and refined by full-matrix

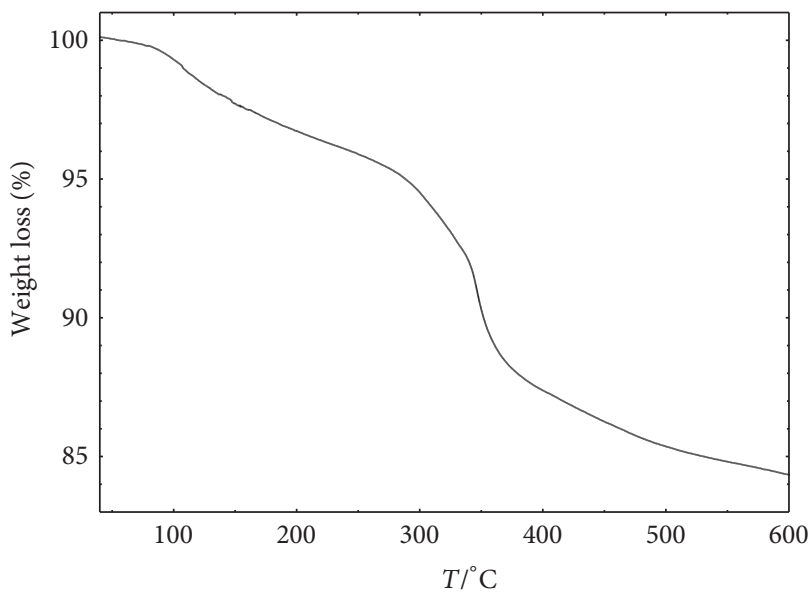

FIgURE 1: TG analyses of I.

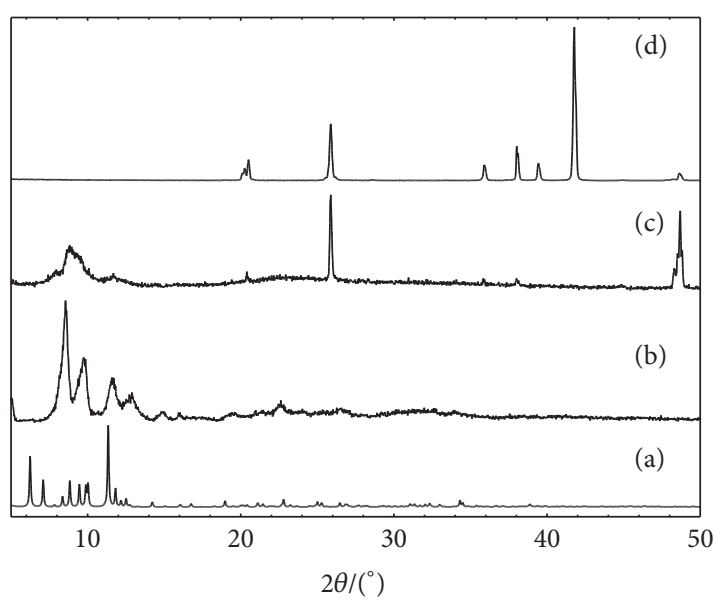

FIGURE 2: The XRD patterns of I. (a) simulated; (b) experimental; (c) treated at $200^{\circ} \mathrm{C}$ for $3 \mathrm{hrs}$; (d) treated at $300^{\circ} \mathrm{C}$ for $3 \mathrm{hrs}$.

least-squares on $F^{2}$ using SHELXL-97. The heavy atoms $(\mathrm{Ge})$ were firstly located by the direct method, and then the light atoms $(\mathrm{N}, \mathrm{O}$, and $\mathrm{F})$ were indentified from the different Fourier maps. All nonhydrogen atoms were refined anisotropically. CCDC 903319 contains the supplementary crystallographic data for this paper. These data can be obtained free of charge from the Cambridge Crystallographic Data Centre via http://www.ccdc.cam.ac.uk/data_request/cif.

A summary of experiment data and refinement parameters of $I$ is given in Table 1. The atomic coordinates and selected bond distances and angles of I are given in Tables 2 and 3 .

2.6. The Solvent Experiments. $10 \mathrm{mg}$ of I was added to $10 \mathrm{~mL}$ water, $10 \mathrm{~mL}$ base $(1 \mathrm{M} \mathrm{NaOH})$, and $10 \mathrm{~mL}$ acid $(1 \mathrm{M} \mathrm{HCl})$ overnight, respectively. The experimental results show that I was unsoluble in the water and soluble in the base and acid. 


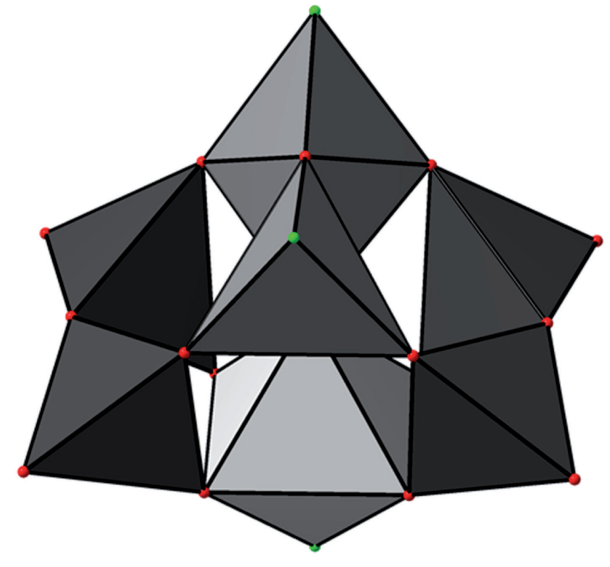

Figure 3: The $\mathrm{Ge}_{7} \mathrm{O}_{14} \mathrm{~F}_{3}$ cluster containing one octahedron, two edge-sharing trigonal bipyramids, and four tetrahedra.

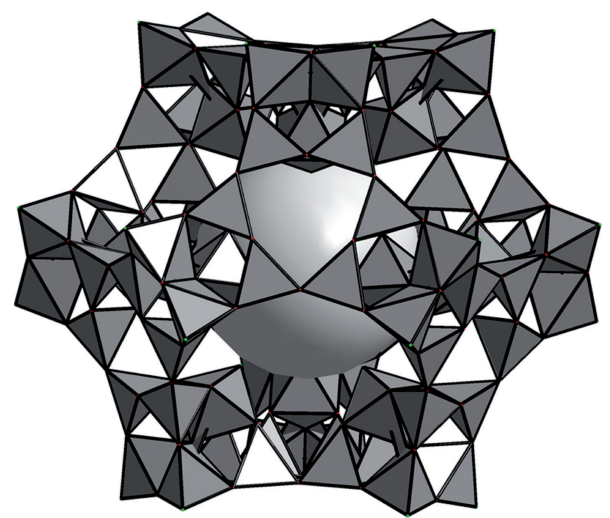

(a)

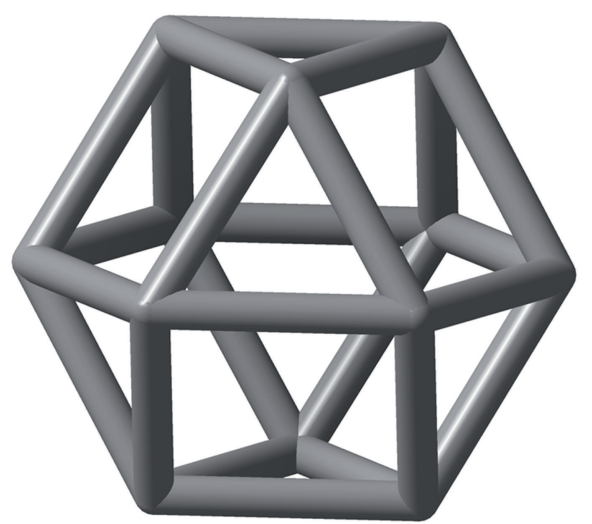

(b)

FIGURE 4: (a) Keggin-type I with the cavity bearing two kinds of apertures defined as $6 \mathrm{MR}$ and $12 \mathrm{MR}$; (b) the simplified I with $\mathrm{Ge}_{7} \mathrm{O}_{14} \mathrm{~F}_{3}$ cluster as node.

\section{Results and Discussion}

I may be designated to the Keggin-type cage of which the $\mathrm{MO}_{6}$ octahedron in $\mathrm{POM}-\mathrm{PMo}_{12} \mathrm{O}_{40}{ }^{3-}$ is replaced with
TABLE 1: The crystallographic data and structural refinement for I.

\begin{tabular}{ll}
\hline Empirical formula & H158 F36 Ge84 N36 O175 \\
Formula weight & 10245.60 \\
Temperature/K & $293(2) \mathrm{K}$ \\
Wavelength/nm & $0.71073 \AA$ \\
Crystal system, space group & Tetragonal, I4/mmm \\
Unit cell dimensions & $a=28.280(4) \AA$ \\
& $c=24.881(5) \AA$ \\
$V /$ nm & $\AA$ \\
$Z, D c /($ Mg.m & -3 \\
$\mu / m^{-1}$ & $19899(6) \AA^{3}$ \\
$F(000)$ & $2,1.632 \mathrm{Mg} / \mathrm{m}^{3}$ \\
Crystal size & 6.309 \\
Range of $\theta$ & 9324 \\
Limiting indices & $0.17 \mathrm{~mm} \times 0.18 \mathrm{~mm} \times 0.20 \mathrm{~mm}$ \\
Reflections collected/unique & $72989 / 4806[R($ int $)=0.2021]$ \\
Completeness to $\theta=25.00^{\circ}$ & $99.3 \%$ \\
Absorption correction & Semiempirical from equivalents \\
Max. and min. transmission & 0.41 and 0.38 \\
Refinement method & Full-matrix least-squares on $F^{2}$ \\
Data/restraints/parameters & $4806 / 0 / 224$ \\
Goodness-of-fit on $F^{2}$ & 1.319 \\
Final $R$ indices $[\mathrm{I}>2 \sigma(\mathrm{I})]$ & $R_{1}=0.0946, w R_{2}=0.2277$ \\
$R$ indices $($ all data $)$ & $R_{1}=0.1638, w R_{2}=0.2514$ \\
Largest diff. peak and hole & 1.970 and $-1.090 \mathrm{e} / \AA^{3}$ \\
\hline$R_{1}=\sum\left\|F_{o}|-| F_{c}\right\| / \sum\left|F_{o}\right| ; w R_{2}=\left\{\sum\left[w\left(F_{o}^{2}-F_{c}^{2}\right)^{2}\right] / \sum\left[w\left(F_{o}^{2}\right)^{2}\right]\right\}^{1 / 2}$. \\
\end{tabular}

$\mathrm{Ge}_{7} \mathrm{O}_{14} \mathrm{~F}_{3}$ cluster. The mixed-coordination $\mathrm{Ge}_{7} \mathrm{O}_{14} \mathrm{~F}_{3}$ cluster has been considerably studied [24-27], which consists of one octahedron, two edge-sharing trigonal bipyramids, and four tetrahedra (Figure 3 ). The distances of $\mathrm{Ge}-\mathrm{O}(1.703(9) \AA 2 \sim 2.168(13) \AA)$ and Ge-F (1.736(14) $\AA$ $1.797(15) \AA$ ) and bond angles of $\mathrm{O}-\mathrm{Ge}-\mathrm{O}\left(88.6(6)^{\circ}\right.$ $\left.178.0(6)^{\circ}\right)$ and $\mathrm{Ge}-\mathrm{O}-\mathrm{Ge}\left(87.5(7)^{\circ} \sim 136.3(3)^{\circ}\right)$ are consistent to the reported results [24].

In Keggin-type $\mathrm{PMo}_{12} \mathrm{O}_{40}{ }^{3-}$ anion cage, twelve $\mathrm{MoO}_{6}$ octahedra can be falled into 4 entities in which three edgesharing $\mathrm{MoO}_{6}$ octahedra are integrated by an oxygen in $\mathrm{PO}_{4}$ tetrahedron. The Keggin cage is formed by vertex-sharingly connecting 4 entities, showing the $T_{d}$ molecular symmetry. In cage of $\mathrm{I}$, twelve $\mathrm{Ge}_{7} \mathrm{O}_{14} \mathrm{~F}_{3}$ clusters surround the cavity of $8.3 \AA$ with two kinds of apertures defined as $6 \mathrm{MR}$ and $12 \mathrm{MR}$. The larger $12 \mathrm{MR}$ aperture gives rise to $5.3 \AA$ pore. Like Keggin $\mathrm{PMo}_{12} \mathrm{O}_{40}^{3-}$ anion, when three $\mathrm{Ge}_{7} \mathrm{O}_{14} \mathrm{~F}_{3}$ clusters by which $6 \mathrm{MR}$ aperture is surrounded are looked as an entity, I mimics the $T_{d}$ Keggin structure (Figure 4 ). In the case of JLG-5 in which the tetrahedral $\mathrm{GeO}_{2}$ units link the adjacent Keggin-type cage into the infinite structure, I becomes the 
TABle 2: Atomic coordinates $\left(\times 10^{4}\right)$ and equivalent isotropic displacement parameters $\left(\AA^{2} \times 10^{3}\right)$ for I.

\begin{tabular}{|c|c|c|c|c|}
\hline Atom & $x$ & $y$ & $z$ & Ueq \\
\hline $\mathrm{Ge}(1)$ & $2343(1)$ & $1584(1)$ & $920(1)$ & $67(1)$ \\
\hline $\mathrm{Ge}(2)$ & $1635(1)$ & 0 & 1913(1) & $64(1)$ \\
\hline $\mathrm{Ge}(3)$ & $1618(1)$ & $1618(1)$ & 0 & $64(1)$ \\
\hline $\mathrm{Ge}(4)$ & $1610(1)$ & $816(1)$ & $2731(1)$ & $67(1)$ \\
\hline $\mathrm{Ge}(5)$ & 2354(1) & $814(1)$ & 1853(1) & $68(1)$ \\
\hline $\mathrm{Ge}(6)$ & 2841(1) & 0 & $2440(1)$ & $76(1)$ \\
\hline $\mathrm{Ge}(7)$ & $2137(1)$ & 0 & $3306(1)$ & $78(1)$ \\
\hline $\mathrm{Ge}(8)$ & $2845(1)$ & $2110(1)$ & 0 & $81(1)$ \\
\hline $\mathrm{F}(1)$ & $1177(4)$ & 0 & $1416(4)$ & $71(3)$ \\
\hline$F(2)$ & $1178(3)$ & $1178(3)$ & 0 & $72(5)$ \\
\hline$F(3)$ & $3461(4)$ & 0 & $2555(6)$ & $97(4)$ \\
\hline $\mathrm{F}(4)$ & $3474(5)$ & $2196(5)$ & 0 & $115(5)$ \\
\hline $\mathrm{F}(5)$ & $2232(6)$ & 0 & $3995(6)$ & $125(5)$ \\
\hline $\mathrm{O}(1)$ & 1302(3) & $470(3)$ & $2294(4)$ & $71(3)$ \\
\hline $\mathrm{O}(2)$ & 2593(3) & $1242(3)$ & $1431(4)$ & $77(3)$ \\
\hline $\mathrm{O}(3)$ & $1251(4)$ & $1251(4)$ & $2997(5)$ & $73(4)$ \\
\hline $\mathrm{O}(4)$ & $1812(4)$ & $536(3)$ & $3311(4)$ & $81(3)$ \\
\hline $\mathrm{O}(5)$ & $2080(3)$ & 1091(3) & $2396(4)$ & $74(3)$ \\
\hline $\mathrm{O}(6)$ & 1945(3) & $1275(3)$ & $530(4)$ & $70(3)$ \\
\hline $\mathrm{O}(7)$ & $2850(3)$ & $1769(3)$ & $592(4)$ & $72(3)$ \\
\hline $\mathrm{O}(8)$ & 2065(3) & 2065(3) & $1236(5)$ & $71(4)$ \\
\hline $\mathrm{O}(9)$ & 2093(4) & 2093(4) & 0 & $64(5)$ \\
\hline $\mathrm{O}(10)$ & $1955(3)$ & $465(3)$ & $1512(4)$ & $67(3)$ \\
\hline $\mathrm{O}(11)$ & $2121(5)$ & 0 & $2435(5)$ & $66(4)$ \\
\hline $\mathrm{O}(12)$ & $2712(5)$ & $2712(5)$ & 0 & $88(7)$ \\
\hline $\mathrm{O}(13)$ & $2867(3)$ & $535(3)$ & 2061(4) & $72(3)$ \\
\hline $\mathrm{O}(14)$ & $2749(5)$ & 0 & $3154(6)$ & $84(4)$ \\
\hline $\mathrm{O}(1 \mathrm{~W})$ & 0 & 0 & 0 & $58(10)$ \\
\hline $\mathrm{O}(2 \mathrm{~W})$ & 0 & 5000 & 0 & $160(19)$ \\
\hline $\mathrm{O}(3 \mathrm{~W})$ & $1542(6)$ & 5000 & 0 & $78(7)$ \\
\hline $\mathrm{N}(1)$ & $474(4)$ & $474(4)$ & $554(6)$ & $60(4)$ \\
\hline $\mathrm{N}(2)$ & $1024(4)$ & $1024(4)$ & $1206(7)$ & $77(5)$ \\
\hline $\mathrm{N}(3)$ & $2517(5)$ & $-359(3)$ & $4395(4)$ & $69(4)$ \\
\hline $\mathrm{N}(4)$ & $1567(9)$ & 0 & $315(12)$ & $63(9)$ \\
\hline
\end{tabular}

Ueq is defined as one third of the trace of the orthogonalized Uij tensor.

OD separated cage because the excessive $\mathrm{HF}$ impedes the formation of $\mathrm{GeO}_{2}$ unit.

\section{Conclusions}

Keggin-type I has been solvothermally obtained. The structural determination shows that in cage of $\mathrm{I}$, twelve $\mathrm{Ge}_{7} \mathrm{O}_{14} \mathrm{~F}_{3}$ clusters surround the cavity of $8.3 \AA$ with two kinds of apertures defined as $6 \mathrm{MR}$ and $12 \mathrm{MR}$. The thermal studies show that Keggin-type cage of I begins to partly collapse from $200^{\circ} \mathrm{C}$ to finally change into $\mathrm{GeO}_{2}$.
TABLE 3: Selected bond lengths $(\AA)$ and bond angles $\left({ }^{\circ}\right)$ for I.

\begin{tabular}{|c|c|c|c|}
\hline $\mathrm{Ge}(1)-\mathrm{O}(6)$ & $1.723(9)$ & $\mathrm{Ge}(5)-\mathrm{O}(10)$ & $1.724(8)$ \\
\hline $\mathrm{Ge}(1)-\mathrm{O}(7)$ & $1.731(9)$ & $\mathrm{Ge}(5)-\mathrm{O}(13)$ & $1.730(9)$ \\
\hline $\mathrm{Ge}(1)-\mathrm{O}(2)$ & $1.747(9)$ & $\mathrm{Ge}(5)-\mathrm{O}(2)$ & $1.738(9)$ \\
\hline $\mathrm{Ge}(1)-\mathrm{O}(8)$ & $1.759(7)$ & $\mathrm{Ge}(5)-\mathrm{O}(5)$ & $1.744(9)$ \\
\hline $\mathrm{Ge}(2)-\mathrm{F}(1)$ & $1.792(10)$ & $\mathrm{Ge}(6)-\mathrm{F}(3)$ & $1.778(12)$ \\
\hline $\mathrm{Ge}(2)-\mathrm{O}(10)^{\mathrm{ii}}$ & $1.881(9)$ & $\mathrm{Ge}(6)-\mathrm{O}(13)^{\mathrm{ii}}$ & $1.785(9)$ \\
\hline $\mathrm{Ge}(2)-\mathrm{O}(10)$ & $1.881(9)$ & $\mathrm{Ge}(6)-\mathrm{O}(13)$ & $1.785(9)$ \\
\hline $\mathrm{Ge}(2)-\mathrm{O}(1)^{\mathrm{ii}}$ & $1.883(10)$ & $\mathrm{Ge}(6)-\mathrm{O}(14)$ & $1.795(15)$ \\
\hline $\mathrm{Ge}(2)-\mathrm{O}(1)$ & $1.883(10)$ & $\mathrm{Ge}(6)-\mathrm{O}(11)$ & $2.034(13)$ \\
\hline $\mathrm{Ge}(2)-\mathrm{O}(11)$ & $1.891(12)$ & $\mathrm{Ge}(7)-\mathrm{F}(5)$ & $1.736(14)$ \\
\hline $\mathrm{Ge}(3)-\mathrm{F}(2)$ & $1.759(14)$ & $\mathrm{Ge}(7)-\mathrm{O}(14)$ & $1.771(16)$ \\
\hline $\mathrm{Ge}(3)-\mathrm{O}(6)^{\mathrm{iii}}$ & $1.880(10)$ & $\mathrm{Ge}(7)-\mathrm{O}(4)^{\mathrm{ii}}$ & $1.774(10)$ \\
\hline $\mathrm{Ge}(3)-\mathrm{O}(6)^{\mathrm{iv}}$ & $1.880(10)$ & $\mathrm{Ge}(7)-\mathrm{O}(4)$ & $1.774(10)$ \\
\hline $\mathrm{Ge}(3)-\mathrm{O}(6)^{\mathrm{i}}$ & $1.880(10)$ & $\mathrm{Ge}(7)-\mathrm{O}(11)$ & $2.168(13)$ \\
\hline $\mathrm{Ge}(3)-\mathrm{O}(6)$ & $1.880(10)$ & $\mathrm{Ge}(8)-\mathrm{O}(12)$ & $1.745(11)$ \\
\hline $\mathrm{Ge}(3)-\mathrm{O}(9)$ & $1.900(17)$ & $\mathrm{Ge}(8)-\mathrm{O}(7)^{\mathrm{iii}}$ & 1.761(9) \\
\hline $\mathrm{Ge}(4)-\mathrm{O}(1)$ & $1.703(9)$ & $\mathrm{Ge}(8)-\mathrm{O}(7)$ & $1.761(9)$ \\
\hline $\mathrm{Ge}(4)-\mathrm{O}(3)$ & $1.728(5)$ & $\mathrm{Ge}(8)-\mathrm{F}(4)$ & $1.797(15)$ \\
\hline $\mathrm{Ge}(4)-\mathrm{O}(4)$ & $1.742(9)$ & $\mathrm{Ge}(8)-\mathrm{O}(9)$ & $2.127(13)$ \\
\hline $\mathrm{Ge}(4)-\mathrm{O}(5)$ & $1.749(9)$ & & \\
\hline $\mathrm{O}(6)-\mathrm{Ge}(1)-\mathrm{O}(7)$ & $115.5(5)$ & $\mathrm{F}(3)-\mathrm{Ge}(6)-\mathrm{O}(13)^{\mathrm{ii}}$ & $92.5(4)$ \\
\hline $\mathrm{O}(6)-\mathrm{Ge}(1)-\mathrm{O}(2)$ & $3.2(5)$ & $\mathrm{F}(3)-\mathrm{Ge}(6)-\mathrm{O}$ & $92.5(4)$ \\
\hline $\mathrm{O}(7)-\mathrm{Ge}(1)-\mathrm{O}(2)$ & $100.1(4)$ & $\mathrm{O}(13)^{\mathrm{ii}}-\mathrm{Ge}(6)$ & $116.0(7)$ \\
\hline $\mathrm{O}(6)-\mathrm{Ge}(1)-\mathrm{O}(8)$ & $110.6(5)$ & $\mathrm{F}(3)-\mathrm{Ge}(6)-\mathrm{O}(14)$ & $89.0(7)$ \\
\hline $\mathrm{O}(7)-\mathrm{Ge}(1)-\mathrm{O}(8)$ & $110.3(5)$ & $\mathrm{O}(13)^{\mathrm{ii}}-\mathrm{Ge}(6)-\mathrm{C}$ & $121.9(3)$ \\
\hline $\mathrm{O}(2)-\mathrm{Ge}(1)-\mathrm{O}(8)$ & $106.4(5)$ & $\mathrm{O}(13)-\mathrm{Ge}(6)-\mathrm{O}(14)$ & $121.9(3)$ \\
\hline $\mathrm{F}(1)-\mathrm{Ge}(2)-\mathrm{O}(10)^{\mathrm{ii}}$ & & $\mathrm{F}(3)-\mathrm{Ge}(6)-\mathrm{O}(11)$ & $171.1(6)$ \\
\hline $\mathrm{F}(1)-\mathrm{Ge}(2)-\mathrm{O}(10)$ & & $\mathrm{O}(13)^{\mathrm{ii}}-\mathrm{Ge}(6)-\mathrm{O}(11)$ & $92.2(4)$ \\
\hline $\mathrm{O}(10)^{\mathrm{ii}}-\mathrm{Ge}(2)-\mathrm{O}(10)$ & $88.6(6)$ & $\mathrm{O}(13)-\mathrm{Ge}(6)-\mathrm{O}(11)$ & $92.2(4)$ \\
\hline $\mathrm{F}(1)-\mathrm{Ge}(2)-\mathrm{O}(1)^{\mathrm{ii}}$ & $89.2(4)$ & $\mathrm{O}(14)-\mathrm{Ge}(6)-\mathrm{O}(11)$ & $82.1(6)$ \\
\hline $\mathrm{O}(10)^{\mathrm{ii}}-\mathrm{Ge}(2)-\mathrm{O}(1)^{\mathrm{ii}}$ & $90.8(4)$ & $\mathrm{F}(5)-\mathrm{Ge}(7)-\mathrm{O}(14)$ & $93.5(8)$ \\
\hline $\mathrm{O}(10)-\mathrm{Ge}(2)-\mathrm{O}(1)^{\mathrm{ii}}$ & $178.0(4)$ & $\mathrm{F}(5)-\mathrm{Ge}(7)-\mathrm{O}(4)^{\mathrm{ii}}$ & $94.2(4)$ \\
\hline $\mathrm{F}(1)-\mathrm{Ge}(2)-\mathrm{O}(1)$ & $89.2(4)$ & $\mathrm{O}(14)-\mathrm{Ge}(7)-\mathrm{O}(4)^{\mathrm{ii}}$ & $120.5(4)$ \\
\hline $\mathrm{O}(10)^{\mathrm{ii}}-\mathrm{Ge}(2)-\mathrm{O}(1)$ & $178.0(4)$ & $\mathrm{F}(5)-\mathrm{Ge}(7)-\mathrm{O}(4)$ & $94.2(4)$ \\
\hline $\mathrm{O}(10)-\mathrm{Ge}(2)-\mathrm{O}(1)$ & $90.8(4)$ & $\mathrm{O}(14)-\mathrm{Ge}(7)-\mathrm{O}(4)$ & $120.5(4)$ \\
\hline $\mathrm{O}(1)^{\mathrm{ii}}-\mathrm{Ge}(2)-\mathrm{O}(1)$ & $89.7(6)$ & $\mathrm{O}(4)^{\mathrm{ii}}-\mathrm{Ge}(7)-\mathrm{O}(4)$ & $117.5(7)$ \\
\hline $\mathrm{F}(1)-\mathrm{Ge}(2)-\mathrm{O}(11)$ & $179.7(6)$ & $\mathrm{F}(5)-\mathrm{Ge}(7)-\mathrm{O}(11)$ & $172.3(7)$ \\
\hline $\mathrm{O}(10)^{\mathrm{ii}}-\mathrm{Ge}(2)-\mathrm{O}(11)$ & $90.9(4)$ & $\mathrm{O}(14)-\mathrm{Ge}(7)-\mathrm{O}(11)$ & $78.8(6)$ \\
\hline $\mathrm{O}(10)-\mathrm{Ge}(2)-\mathrm{O}(11)$ & $90.9(4)$ & $\mathrm{O}(4)^{\mathrm{ii}}-\mathrm{Ge}(7)-\mathrm{O}(11)$ & $89.8(4)$ \\
\hline $\mathrm{O}(1)^{\mathrm{ii}}-\mathrm{Ge}(2)-\mathrm{O}(11)$ & $91.0(4)$ & $\mathrm{O}(4)-\mathrm{Ge}(7)-\mathrm{O}(11)$ & $89.8(4)$ \\
\hline $\mathrm{O}(1)-\mathrm{Ge}(2)-\mathrm{O}(11)$ & $91.0(4)$ & $\mathrm{O}(12)-\mathrm{Ge}(8)-\mathrm{O}(7)^{\mathrm{iii}}$ & $122.5(3)$ \\
\hline $\mathrm{F}(2)-\mathrm{Ge}(3)-\mathrm{O}(6)^{\mathrm{iii}}$ & $89.0(3)$ & $\mathrm{O}(12)-\mathrm{Ge}(8)-\mathrm{O}(7)$ & $122.5(3)$ \\
\hline $\mathrm{F}(2)-\mathrm{Ge}(3)-\mathrm{O}(6)^{\mathrm{iv}}$ & $89.0(3)$ & $\mathrm{O}(7)^{\mathrm{iii}}-\mathrm{Ge}(8)-\mathrm{O}(7)$ & $113.5(7)$ \\
\hline $\mathrm{O}(6)^{\mathrm{iii}}-\mathrm{Ge}(3)-\mathrm{O}(6)^{\mathrm{iv}}$ & $90.9(6)$ & $\mathrm{O}(12)-\mathrm{Ge}(8)-\mathrm{F}(4)$ & $94.6(7)$ \\
\hline $\mathrm{F}(2)-\mathrm{Ge}(3)-\mathrm{O}(6)^{\mathrm{i}}$ & $89.0(3)$ & $\mathrm{O}(7)^{\mathrm{iii}}-\mathrm{Ge}(8)-\mathrm{F}(4)$ & $93.8(4)$ \\
\hline $\mathrm{O}(6)^{\mathrm{iii}}-\mathrm{Ge}(3)-\mathrm{O}(6)^{\mathrm{i}}$ & $178.0(6)$ & $\mathrm{O}(7)-\mathrm{Ge}(8)-\mathrm{F}(4)$ & $93.8(4)$ \\
\hline $\mathrm{O}(6)^{\mathrm{iv}}-\mathrm{Ge}(3)-\mathrm{O}(6)^{\mathrm{i}}$ & $89.1(6)$ & $\mathrm{O}(12)-\mathrm{Ge}(8)-\mathrm{O}(9)$ & $78.8(6)$ \\
\hline $\mathrm{F}(2)-\mathrm{Ge}(3)-\mathrm{O}(6)$ & $89.0(3)$ & $\mathrm{O}(7)^{\mathrm{iii}}-\mathrm{Ge}(8)-\mathrm{O}(9)$ & $89.8(3)$ \\
\hline $\mathrm{O}(6)^{\mathrm{iii}}-\mathrm{Ge}(3)-\mathrm{O}(6)$ & $89.1(6)$ & $\mathrm{O}(7)-\mathrm{Ge}(8)-\mathrm{O}(9)$ & $89.8(3)$ \\
\hline $\mathrm{O}(6)^{\mathrm{iv}}-\mathrm{Ge}(3)-\mathrm{O}(6)$ & $178.0(6)$ & $\mathrm{F}(4)-\mathrm{Ge}(8)-\mathrm{O}(9)$ & $173.5(6)$ \\
\hline $\mathrm{O}(6)^{\mathrm{i}}-\mathrm{Ge}(3)-\mathrm{O}(6)$ & $90.9(6)$ & $\mathrm{Ge}(4)-\mathrm{O}(1)-\mathrm{Ge}(2)$ & $118.1(5)$ \\
\hline
\end{tabular}


TABLE 3: Continued.

\begin{tabular}{lclc}
\hline $\mathrm{F}(2)-\mathrm{Ge}(3)-\mathrm{O}(9)$ & $180.0(7)$ & $\mathrm{Ge}(5)-\mathrm{O}(2)-\mathrm{Ge}(1)$ & $131.9(5)$ \\
$\mathrm{O}(6)^{\mathrm{iii}}-\mathrm{Ge}(3)-\mathrm{O}(9)$ & $91.0(3)$ & $\mathrm{Ge}(4)^{\mathrm{i}}-\mathrm{O}(3)-\mathrm{Ge}(4)$ & $133.6(8)$ \\
$\mathrm{O}(6)^{\mathrm{iv}}-\mathrm{Ge}(3)-\mathrm{O}(9)$ & $91.0(3)$ & $\mathrm{Ge}(4)-\mathrm{O}(4)-\mathrm{Ge}(7)$ & $123.5(6)$ \\
$\mathrm{O}(6)^{\mathrm{i}}-\mathrm{Ge}(3)-\mathrm{O}(9)$ & $91.0(3)$ & $\mathrm{Ge}(5)-\mathrm{O}(5)-\mathrm{Ge}(4)$ & $120.5(5)$ \\
$\mathrm{O}(6)-\mathrm{Ge}(3)-\mathrm{O}(9)$ & $91.0(3)$ & $\mathrm{Ge}(1)-\mathrm{O}(6)-\mathrm{Ge}(3)$ & $117.0(5)$ \\
$\mathrm{O}(1)-\mathrm{Ge}(4)-\mathrm{O}(3)$ & $110.8(6)$ & $\mathrm{Ge}(1)-\mathrm{O}(7)-\mathrm{Ge}(8)$ & $123.5(5)$ \\
$\mathrm{O}(1)-\mathrm{Ge}(4)-\mathrm{O}(4)$ & $115.8(5)$ & $\mathrm{Ge}(1)-\mathrm{O}(8)-\mathrm{Ge}(1)^{\mathrm{i}}$ & $119.5(8)$ \\
$\mathrm{O}(3)-\mathrm{Ge}(4)-\mathrm{O}(4)$ & $101.4(5)$ & $\mathrm{Ge}(3)-\mathrm{O}(9)-\mathrm{Ge}(8)^{\mathrm{iv}}$ & $136.3(3)$ \\
$\mathrm{O}(1)-\mathrm{Ge}(4)-\mathrm{O}(5)$ & $109.8(5)$ & $\mathrm{Ge}(3)-\mathrm{O}(9)-\mathrm{Ge}(8)$ & $136.3(3)$ \\
$\mathrm{O}(3)-\mathrm{Ge}(4)-\mathrm{O}(5)$ & $108.2(6)$ & $\mathrm{Ge}(8)-\mathrm{O}(9)-\mathrm{Ge}(8)$ & $87.5(7)$ \\
$\mathrm{O}(4)-\mathrm{Ge}(4)-\mathrm{O}(5)$ & $110.3(5)$ & $\mathrm{Ge}(5)-\mathrm{O}(10)-\mathrm{Ge}(2)$ & $117.0(5)$ \\
$\mathrm{O}(10)-\mathrm{Ge}(5)-\mathrm{O}(13)$ & $115.8(4)$ & $\mathrm{Ge}(2)-\mathrm{O}(11)-\mathrm{Ge}(6)$ & $137.0(7)$ \\
$\mathrm{O}(10)-\mathrm{Ge}(5)-\mathrm{O}(2)$ & $110.9(4)$ & $\mathrm{Ge}(2)-\mathrm{O}(11)-\mathrm{Ge}(7)$ & $134.5(7)$ \\
$\mathrm{O}(13)-\mathrm{Ge}(5)-\mathrm{O}(2)$ & $99.9(4)$ & $\mathrm{Ge}(6)-\mathrm{O}(11)-\mathrm{Ge}(7)$ & $88.5(5)$ \\
$\mathrm{O}(10)-\mathrm{Ge}(5)-\mathrm{O}(5)$ & $110.3(4)$ & $\mathrm{Ge}(8)-\mathrm{O}(12)-\mathrm{Ge}(8)^{\mathrm{iv}}$ & $114.9(11)$ \\
$\mathrm{O}(13)-\mathrm{Ge}(5)-\mathrm{O}(5)$ & $110.2(5)$ & $\mathrm{Ge}(5)-\mathrm{O}(13)-\mathrm{Ge}(6)$ & $120.6(5)$ \\
$\mathrm{O}(2)-\mathrm{Ge}(5)-\mathrm{O}(5)$ & $109.1(5)$ & $\mathrm{Ge}(7)-\mathrm{O}(14)-\mathrm{Ge}(6)$ & $110.6(7)$ \\
\hline
\end{tabular}

Symmetry transformations used to generate equivalent atoms: ${ }^{\mathrm{i}} y, x, z$; ${ }^{\text {ii }} x,-y, z ;{ }^{\text {iii }} x, y,-z ;{ }^{\text {iv }} y, x,-z$.

\section{Acknowledgment}

This work was supported by the Scientific Research Foundation for the Returned Overseas Team, Chinese Education Ministry.

\section{References}

[1] D. W. Breck, Zeolite Molecular Sieves: Structure, Chemistry and Use, Wiley \& Sons, New York, NY, USA, 1974.

[2] J. Cejka, A. Corma, and S. Zones, Zeolites and Catalysis: Synthesis, Reactions and Applications, Wiley-VCH, Weinheim, Germany, 2010.

[3] G. O. Brunner and W. M. Meier, "Framework density distribution of zeolite-type tetrahedral nets," Nature, vol. 337, no. 6203, pp. 146-147, 1989.

[4] M. O’Keeffe and O. M. Yaghi, "Germanate zeolites: contrasting the behavior of germanate and silicate structures built from cubic $\mathrm{T}_{8} \mathrm{O}_{20}$ units ( $\mathrm{T}=\mathrm{Ge}$ or $\mathrm{Si}$ ), Chemistry, vol. 5, no. 10, pp. 2796-2801, 1999.

[5] J. Jiang, J. L. Jorda, M. J. Diaz-Cabanas, J. Yu, and A. Corma, "The synthesis of an extra-large-pore zeolite with double threering building units and a low framework density," Angewandte Chemie, vol. 49, no. 29, pp. 4986-4988, 2010.

[6] T. Conradsson, M. S. Dadachov, and X. D. Zou, "Synthesis and structure of $\left(\mathrm{Me}_{3} \mathrm{~N}\right)_{6}\left[\mathrm{Ge}_{32} \mathrm{O}_{64}\right] \cdot\left(\mathrm{H}_{2} \mathrm{O}\right)_{4.5}$, a thermally stable novel zeotype with 3D interconnected 12-ring channels," Microporous and Mesoporous Materials, vol. 41, no. 1-3, pp. 183-191, 2000.

[7] H. Li and O. M. Yaghi, "Transformation of germanium dioxide to microporous germanate 4-connected nets," Journal of the American Chemical Society, vol. 120, no. 40, pp. 10569-10570, 1998.

[8] Y. Mathieu, J.-L. Paillaud, P. Caullet, and N. Bats, "Synthesis and characterization of IM-10: a new microporous silicogermanate with a novel topology," Microporous and Mesoporous Materials, vol. 75, no. 1-2, pp. 13-22, 2004.
[9] J. Su, Y. Wang, Z. Wang, and J. Lin, "PKU-9: an aluminogermanate with a new three-dimensional zeolite framework constructed from CGS layers and spiro-5 units," Journal of the American Chemical Society, vol. 131, no. 17, pp. 6080-6081, 2009.

[10] A. Corma, M. J. Diaz-Cabanas, J. L. Jorda, F. Rey, G. Sastre, and K. G. Strohmaier, "A zeolitic structure (ITQ-34) with connected 9- and 10-ring channels obtained with phosphonium cations as structure directing agents," Journal of the American Chemical Society, vol. 130, no. 49, pp. 16482-16483, 2008.

[11] Y. Lorgouilloux, M. Dodin, J. L. Paillaud et al., "IM-16: a new microporous germanosilicate with a novel framework topology containing $d 4 r$ and $m t w$ composite building units," Journal of Solid State Chemistry, vol. 182, no. 3, pp. 622-629, 2009.

[12] X. Bu, P. Feng, T. E. Gier, D. Zhao, and G. D. Stucky, "Hydrothermal synthesis and structural characterization of zeolite-like structures based on gallium and aluminum germanates," Journal of the American Chemical Society, vol. 120, no. 51, pp. 13389-13397, 1998.

[13] D. L. Dorset, K. G. Strohmaier, C. E. Kliewer et al., "Crystal structure of ITQ-26, a 3D framework with extra-large pores," Chemistry of Materials, vol. 20, no. 16, pp. 5325-5331, 2008.

[14] L. Tang, L. Shi, C. Bonneau et al., "A zeolite family with chiral and achiral structures built from the same building layer," Nature Materials, vol. 7, no. 5, pp. 381-385, 2008.

[15] T. E. Gier, X. Bu, P. Feng, and G. D. Stucky, "Synthesis and organization of zeolite-like materials with three-dimensional helical pores," Nature, vol. 395, no. 6698, pp. 154-157, 1998.

[16] J. Sun, C. Bonneau, A. Cantin et al., “The ITQ-37 mesoporous chiral zeolite," Nature, vol. 458, no. 7242, pp. 1154-1157, 2009.

[17] Y. F. Li and X. D. Zou, "SU-16: a three-dimensional openframework borogermanate with a novel zeolite topology," Angewandte Chemie, vol. 44, no. 13, pp. 2012-2015, 2005.

[18] X. Zou, T. Conradsson, M. Klingstedt, M. S. Dadachov, and M. O'Keeffe, "A mesoporous germanium oxide with crystalline pore walls and its chiral derivative," Nature, vol. 437, no. 7059, pp. 716-719, 2005.

[19] M. V. Peskov and X. Zou, "Germanates built from $\mathrm{Ge}_{10}(\mathrm{O}$, $\mathrm{OH})_{27-28}$ and $\mathrm{Ge}_{7}(\mathrm{O}, \mathrm{OH}, \mathrm{F})_{19}$ secondary building units: from systematic study of reported compounds to rational design of novel structures," Journal of Physical Chemistry C, vol. 115, no. 15, pp. 7729-7739, 2011.

[20] B. Guo, A. K. Inge, C. Bonneau et al., "Investigation of the $\mathrm{GeO}_{2}$-1,6-diaminohexane-water-pyridine-HF phase diagram leading to the discovery of two novel layered germanates with extra-large rings," Inorganic Chemistry, vol. 50, no. 1, pp. 201-207, 2011.

[21] C. Bonneau, J. Sun, R. Sanchez-Smith et al., "Open-framework germanate built from the hexagonal packing of rigid cylinders," Inorganic Chemistry, vol. 48, no. 21, pp. 9962-9964, 2009.

[22] Q. Pan, J. Li, K. E. Christensen et al., "A germanate built from a $6^{8} 12^{6}$ cavity cotemplated by an $\left(\mathrm{H}_{2} \mathrm{O}\right)_{16}$ cluster and 2-methylpiperazine," Angewandte Chemie, vol. 47, no. 41, pp. 7868-7871, 2008.

[23] Y. Han, Y. Li, J. Yu, and R. R. Xu, "A gallogermanate zeolite constructed exclusively by three-ring building units," Angewandte Chemie, vol. 50, no. 13, pp. 3003-3005, 2011.

[24] J. Plévert, T. M. Gentz, T. L. Groy, M. O’Keeffe, and O. M. Yaghi, "Layered structures constructed from new linkages of $\mathrm{Ge}_{7}(\mathrm{O}, \mathrm{OH}, \mathrm{F})_{19}$ clusters," Chemistry of Materials, vol. 15, no. 3, pp. 714-718, 2003. 
[25] H. Li, M. Eddaoudi, D. A. Richardson, and O. M. Yaghi, "Porous germanates: synthesis, structure, and inclusion properties of $\mathrm{Ge}_{7} \mathrm{O}_{14.5} \mathrm{~F}_{2} \cdot\left[\left(\mathrm{CH}_{3}\right)_{2} \mathrm{NH}_{2}\right]_{3}\left(\mathrm{H}_{2} \mathrm{O}\right)_{0.86}$," Journal of the American Chemical Society, vol. 120, no. 33, pp. 8567-8568, 1998.

[26] J. Plévert, T. M. Gentz, A. Laine et al., "A flexible germanate structure containing 24-ring channels and with very low framework density," Journal of the American Chemical Society, vol. 123, no. 50, pp. 12706-12707, 2001.

[27] L. Shi, C. Bonneau, Y. Li, J. Sun, H. Yue, and X. Zou, "SU-22 and SU-23: layered germanates built from 4-coordinated $\mathrm{Ge}_{7}$ Clusters exhibiting structural variations on the 44 topology," Crystal Growth \& Design, vol. 8, no. 10, pp. 3695-3699, 2008. 

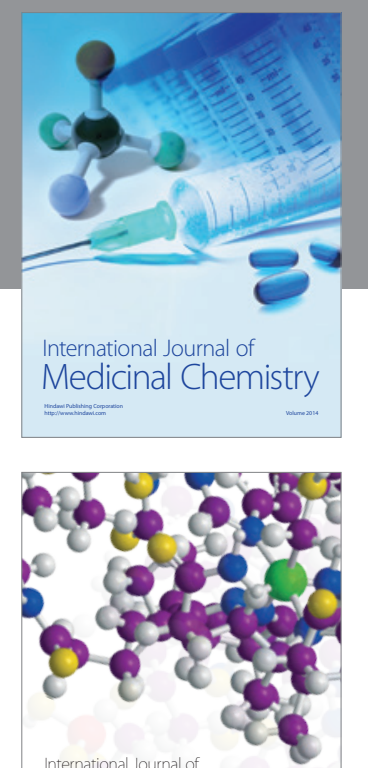

\section{Carbohydrate} Chemistry

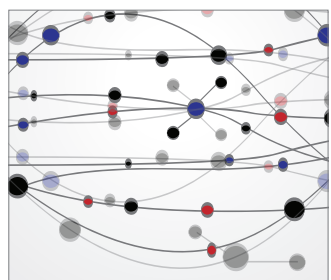

The Scientific World Journal
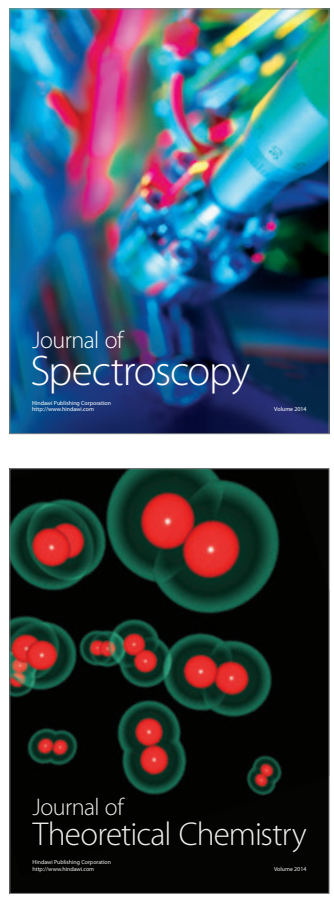
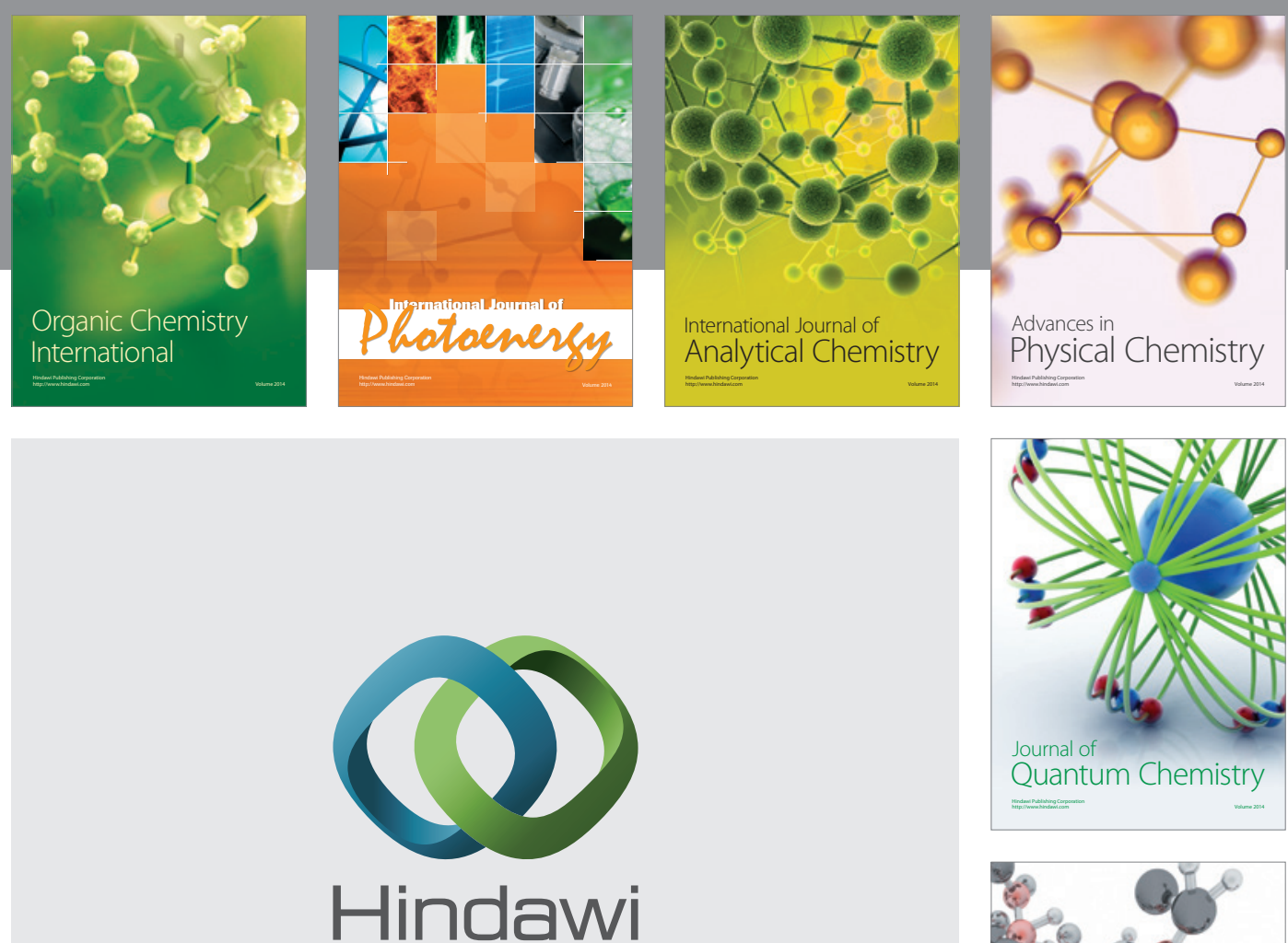

Submit your manuscripts at

http://www.hindawi.com

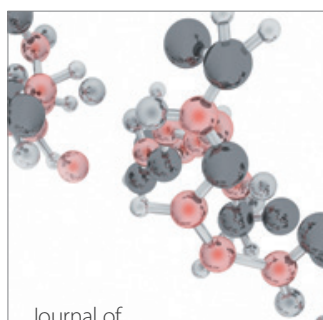

Analytical Methods

in Chemistry

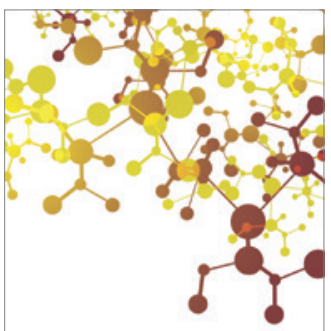

Journal of

Applied Chemistry

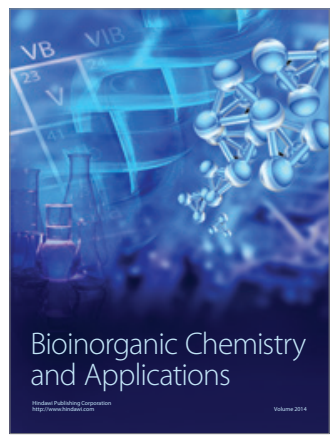

Inorganic Chemistry
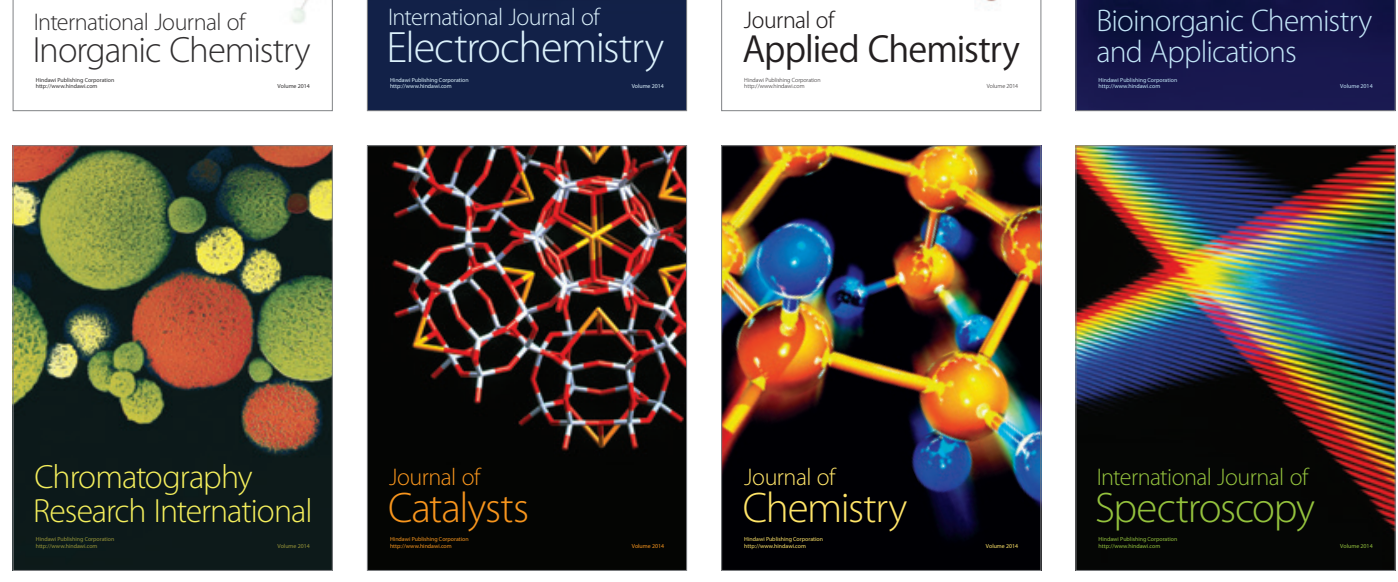